\title{
Síndrome antifosfolipídico en pediatría: a propósito de un caso clínico
}

\author{
Antiphospholipid syndrome in Pediatrics: a case report
}

\author{
Dra. Eugenia Micheletti ${ }^{a}$ Dra. Luciana Blanco ${ }^{a}$ Dra. Ana Cechinni ${ }^{a}$, Dr. Marcelo Acerenza $a^{a}$ y Dra. Paula Canda ${ }^{a}$
}

\begin{abstract}
RESUMEN
Elsíndromeantifosfolipídicoesunaenfermedadautoinmunitaria en la cual se producen de forma persistente autoanticuerpos contra una variedad de fosfolípidos y proteínas transportadoras de estos. Ocurre en el 1,8\% de la población y solo el $2 \%$ de los casos son pediátricos.

El espectro de manifestaciones clínicas es amplio: desde pacientes asintomáticos hasta una enfermedad amenazante para la vida como es el síndrome antifosfolipídico catastrófico. Cualquier órgano puede verse afectado como consecuencia de la trombosis a nivel de los grandes vasos o la microcirculación. Las manifestaciones más frecuentes en pediatría corresponden a trombosis venosas en el $60 \%$ de los pacientes, trombosis arterial en el $32 \%$, alteraciones hematológicas en el $38 \%$ (plaquetopenia, leucopenia), alteraciones en la piel en el 18\% (livedo reticularis, fenómeno de Raynaud) y alteraciones neurológicas en el $16 \%$. Se presenta el caso clínico de una paciente pediátrica por la baja incidencia a esta edad.

Palabras clave: síndrome antifosfolipídico, pediatría.
\end{abstract}

\section{ABSTRACT}

The antiphospholipid syndrome is a multisystem autoimmune disease in which autoantibodies against a variety of phospholipids and phospholipid binding proteins are produced. It occurs in $1.8 \%$ of the population and only $2 \%$ of the cases are pediatric.

The spectrum of clinical manifestations is wide from asymptomatic patients to a life-threatening disease like the catastrophic antiphospholipid syndrome. Any organ can be affected. The most frequent manifestations in pediatrics correspond to venous thrombosis in $60 \%$ of patients, arterial thrombosis in 32\%, hematological disease in 38\% (thrombocytopenia, leucopenia), skin alterations in $18 \%$ (livedo reticularis, Raynaud's phenomenon) and neurological signs in $16 \%$.

We describe the case of a previously healthy 14 -year-old female patient diagnosed with antiphospholipid syndrome.

Key words: antiphospholipid syndrome, pediatrics.

http:/ / dx.doi.org/10.5546/ aap.2017.e412

Cómo citar: Micheletti, Blanco L, Cechinni A, et al. Síndrome antifosfolipídico en pediatría: a propósito de un caso clínico. Arch Argent Pediatr 2017;115(6):e412-e415.

a. Sanatorio Sagrado Corazón.

Ciudad Autónoma de Buenos Aires.

Correspondencia: Dra. Paula Canda: paulacanda@hotmail.com

Financiamiento: Ninguno.

Conflicto de intereses: Ninguno que declarar.

Recibido: 4-11-2016

Aceptado: 13-6-2017

\section{INTRODUCCIÓN}

El síndrome antifosfolipídico (SAF) es una enfermedad autoinmunitaria en la cual se producen de forma persistente autoanticuerpos contra una variedad de fosfolípidos y proteínas transportadoras de estos. Ocurre en el 1,8\% de la población y solo el $2 \%$ de los casos son pediátricos. Se caracteriza por la aparición de trombosis repetidas (tanto venosas como arteriales), morbilidad en los embarazos (abortos recurrentes) y alteraciones hematológicas (trombocitopenia o anemia hemolítica) asociados a la presencia de anticuerpos antifosfolipídicos (anticardiolipinas, anticoagulante lúpico y antiB2-glucoproteína -B2GP1-) y a desórdenes protrombóticos congénitos, como mutaciones en el factor $\mathrm{V}$ de Leiden y deficiencia de proteínas $\mathrm{S}$ y C y antitrombina III. ${ }^{1,2}$

Este síndrome puede ser primario o asociado a otra patología autoinmune subyacente, principalmente, al lupus eritematoso sistémico (LES) (Tabla 1).

El espectro clínico de presentación es muy variado, ya que puede afectar cualquier órgano o sistema. En pediatría, al inicio del cuadro clínico, el $60 \%$ de los pacientes presentaron trombosis venosa (miembros inferiores, seno venoso cerebral, vena porta), trombosis arterial el $32 \%$ (accidente cerebrovascular $-\mathrm{ACV}-$ ),

TABLA 1. Enfermedades sistémicas asociadas a sindrome antifosfolipídico

- Lupus eritematoso sistémico

- Artritis idiopática juvenil

- Polimialgia reumática

- Esclerodermia

- Poliarteritis nodosa

- Policondritis recidivante

- Arteritis de células gigantes

- Arteritis de Takayasu

- Síndrome de Evans

- Anemia hemolítica autoinmune

- Púrpura trombocitopénica autoinmune

- Síndrome de Budd-Chiari

- Trombosis del seno venoso central

* Tabla de elaboración propia. 
alteraciones hematológicas el 38\% (síndrome de Evans, trombocitopenia, leucopenia, anemia hemolítica), alteraciones en la piel (livedo reticularis, fenómeno de Raynaud) el $18 \%$ y alteraciones neurológicas (migraña, corea/ atetosis, epilepsia) el $16 \%$.

Esta entidad clínica se considera el estado más común de hipercoagulabilidad adquirida y ocurre en menos del 1,8\% de la población general y solo el $2 \%$ de dicha población tiene menos de 15 años al momento del diagnóstico. La proporción mujerhombre es de 1,2:1 en pediatría y 5:1 en adultos. ${ }^{2}$

Existen diferentes criterios clínicos y de laboratorio para el diagnóstico de SAF, que se describen en la Tabla $2 .^{3-6}$

Una variante aguda del síndrome (SAF catastrófico) conduce a una microangiopatía trombótica masiva e insuficiencia multiorgánica ${ }^{3}$ (Tabla 3).

La tomografía axial computada (TAC) helicoidal angiográfica pulmonar es el estándar de oro para el diagnóstico por imágenes, con una sensibilidad del $100 \%$ y una especificidad del $96 \% .^{7}$

Se presenta el caso clínico de una paciente pediátrica por la baja incidencia a esta edad.

\section{CASO CLÍNICO}

Paciente de sexo femenino de 14 años de edad previamente sana que consultó en la Guardia por presentar un episodio sincopal asociado a palpitaciones y vómitos.

En su ingreso, se encontraba hemodinámicamente compensada, con palidez cutáneo-mucosa, taquipnea, taquicardia y refería dolor preesternal, sin otros datos para destacar.

Se realizó un análisis de laboratorio, en el que se observó como dato positivo plaquetopenia: $39000 \mathrm{~mm}^{3}$; dímero D con valor elevado: $7494 \mathrm{ng}$ / $\mathrm{ml}$ (valor normal hasta $500 \mathrm{ng} / \mathrm{ml}$ ); y fibrinógeno normal: $330 \mathrm{mg} / \mathrm{dl}$.

Por disnea progresiva y ante la sospecha de tromboembolismo de pulmón (TEP), se realizó una TAC de tórax, que evidenció tromboembolismo pulmonar, que afectaba ambas arterias pulmonares y sus ramas, mínimo derrame pericárdico y parénquima pleuropulmonar sin alteraciones (Figura 1).

En el electrocardiograma realizado, se evidenció taquicardia sinusal, intervalo QTc en el límite superior $(0,48 \mathrm{~s})$ y onda $\mathrm{T}$ invertida, que se interpretaron como alteraciones secundarias a la isquemia.

TABLA 2. Criterios diagnósticos para el síndrome antifosfolipídico

\section{Criterios clínicos}

- Trombosis vascular: uno o más episodios clínicos de trombosis arterial o venosa de pequeños vasos en cualquier órgano.

- Morbilidad en el embarazo.

\section{Criterios de laboratorio}

- Anticuerpo anticardiolipina en suero o plasma IgG o IgM (valor mayor de 40 unidades por ensayo por inmunoabsorción ligado a enzimas -enzyme-linked immunosorbent assay; ELISA, por sus siglas en inglés-) en dos determinaciones separadas por 12 semanas.

- Anticuerpo anti-B2-glucoproteína IgG o IgM positivos en dos determinaciones con diferencia de 12 semanas.

- Factor anticoagulante lúpico positivo.

- También se observan, en el $45 \%$ de los pacientes, desórdenes protrombóticos congénitos, como mutaciones del factor $\mathrm{V}$ de Leiden o alteraciones en las proteínas $\mathrm{S}$ y $\mathrm{C}$.

El diagnóstico se estable con, al menos, un criterio clínico mayor y una prueba confirmatoria positiva.

* Tabla de elaboración propia.

TABLA 3. Criterios diagnósticos de síndrome antifosfolipídico catastrófico

- Evidencia de afectación de tres o más órganos, sistemas o tejidos.

- Aparición de las manifestaciones simultáneamente en menos de una semana.

- Confirmación histológica de obstrucción de pequeños vasos en, al menos, un órgano o tejido.

- Confirmación en el laboratorio de presencia de anticuerpos antifosfolipídicos.

* Tabla de elaboración propia. 
Se realizó un ecodoppler de los vasos del cuello y los miembros inferiores, que informó: "Ambas venas yugulares permeables, al igual que las venas femorales derechas. Vena femoral común izquierda y vena femoral superficial proximal izquierda con contenido hipoecoico, no compresible. Sin señal doppler color en su interior (trombosis), vena cava inferior permeable".

Se realizó un ecocardiograma, que informó: "Derrame pericárdico anterior y posterior escaso con ligera dilatación de cavidades derechas, hipertensión pulmonar leve de $40 \mathrm{mmHg}$ con función de ventrículo izquierdo (VI) y ventrículo derecho (VD) conservada y coronarias normales".

Se solicitó perfil reumatológico que incluía reactantes de fase aguda $\mathrm{C} 3, \mathrm{C} 4, \mathrm{CH} 50$ normales y factor antinuclear (FAN), factor reumatoideo (FR), anti-ADN negativos.

Se realizó un análisis de laboratorio con sospecha de síndrome protrombótico con anticuerpos anticoagulante lúpico positivo fuerte, beta-2-microglobulina IgG 88 (positivo mayor de

FIgURA 1. Tomografía de tórax
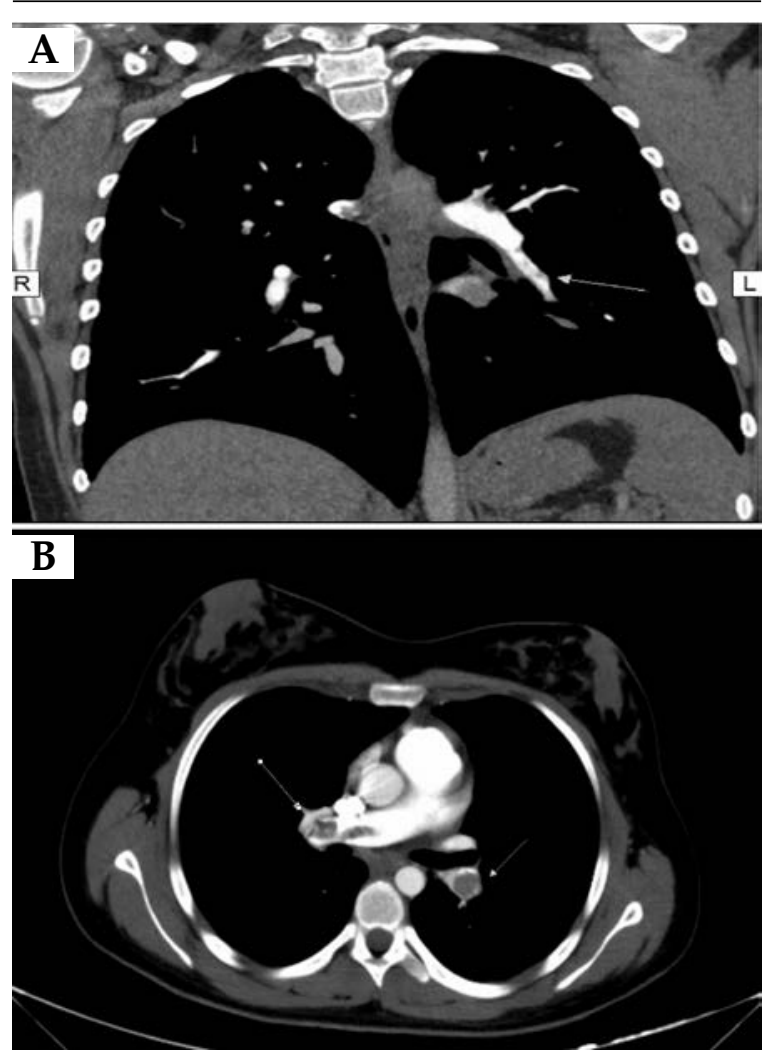

A. Tromboembolismo pulmonar que afecta a ambas arterias pulmonares y sus ramas. B. Mínimo derrame pericárdico. Parénquima pulmonar sin alteraciones.
20), anticuerpos anticardiolipina IgG 80 positivo. Estos tres marcadores se repitieron en forma ambulatoria a las 12 semanas y continuaron positivos.

Factor V de Leiden, proteína C, proteína $S$, productos de degradación del fibrinógeno (PDF), protrombina 20210A, metilentetrahidrofolato reductasa (MTHR): normales.

Inició anticoagulación con diagnóstico de SAF y realizó en forma secuencial enoxaparina / acenocumarol.

La paciente continúa, actualmente, en control con el pediatra de cabecera y la hematóloga, con buen manejo de la anticoagulación y asintomática.

\section{DISCUSIÓN}

El SAF es una enfermedad autoinmune, en la que se altera la coagulación sanguínea y se caracteriza por episodios trombóticos esporádicos impredecibles y, en ocasiones, con compromiso vital.

Se considera, actualmente, como una de las principales y más frecuentes diátesis protrombóticas.

Su primera descripción fue realizada en la década del ochenta. En los últimos años, se han incrementado las publicaciones de casos que dan información sobre el espectro de esta enfermedad. ${ }^{8}$

Ocurre, principalmente, en mujeres jóvenes en edad fértil. Su causa es desconocida, pero se plantea que es multifactorial. Se cree que ciertos factores genéticos relacionados con las moléculas HLA-DR7, HLA-DR4, HLA DRW 53 podrían jugar un papel fundamental en la presentación de este síndrome y también mutaciones del factor $\mathrm{V}$ de Leiden y deficiencias de antitrombina III, proteína $\mathrm{C}$ y proteína $\mathrm{S}$.

Los mecanismos y la fisiopatología de los síntomas clínicos vinculados con el SAF son extremadamente heterogéneos. ${ }^{1,2}$

Los anticuerpos con actividad anticoagulante lúpica en los pacientes con SAF están dirigidos contra la B2GP1 o contra la protrombina. Estos anticuerpos se fijan a la protrombina y se unen a los fosfolípidos aniónicos de la membrana celular y desplazan factores de coagulación. ${ }^{3}$

El SAF, en su forma primaria, puede ser visto como una enfermedad autoinmune organoespecífica, con anticuerpos dirigidos contra un pequeño número de proteínas plasmáticas y antígenos relacionados. Además, el SAF y los mismos anticuerpos pueden ocurrir como un síndrome secundario en el marco de un LES u otras enfermedades autoinmunes. ${ }^{3,9}$ 
La prevalencia de anticuerpos anticardiolipina o anticoagulante lúpico observados en pacientes pediátricos con LES oscila entre 30-70\% y $10-40 \%$, respectivamente. La frecuencia del SAF en niños con LES se estima de hasta el $50 \%$.

Una variante aguda de este síndrome es el "SAF catastrófico", que ocurre en menos del 1\% de todos los pacientes afectados y conduce a microangiopatía trombótica masiva e insuficiencia multiorgánica en más de la mitad de los casos, que puede presentar una evolución fatal.

Se establecieron criterios para su diagnóstico según el consenso internacional de Sídney (2006). ${ }^{10}$ Se consideran criterios mayores trombosis venosa o arterial, aborto espontáneo, trombocitopenia. Otros criterios clínicos son alteración de válvulas cardíacas, prueba de Coombs positiva, livedo reticularis, migraña, úlceras en las piernas, corea, necrosis avascular. ${ }^{1}$

Las pruebas confirmatorias se basan en anticuerpos anticardiolipinas tipo IgG $>20$ unidades, $\operatorname{IgM}>20$ unidades, prueba de anticoagulante lúpico positiva. ${ }^{1,3}$

El diagnóstico se establece únicamente cuando existe, al menos, un criterio clínico mayor y, por lo menos, una prueba confirmatoria positiva. ${ }^{1,3}$

El tratamiento consiste, en primer lugar, en eliminar o reducir aquellos factores de riesgo vascular en estos pacientes (hipertensión arterial, hipercolesterolemia, tabaquismo, anticonceptivos orales que contengan estrógenos, obesidad, inmovilización). Debe iniciarse profilaxis en pacientes asintomáticos con antiagregantes (ácido acetilsalicílico, AAS) o anticoagulantes dicumarínicos, y aquellos pacientes con trombosis deben recibir heparina y luego, con el monitoreo adecuado, rotar a acenocumarol.

El tratamiento del SAF catastrófico consiste, además de la anticoagulación con heparina de inicio, en el agregado de metilprednisolona endovenosa.

En diferentes series de casos pediátricos, ${ }^{4,11,12}$ la media de edad en esta población es de 15,3 años; las trombosis venosas fueron la manifestación más frecuente presentes en el $64,7 \%$ de los pacientes y, dentro de este grupo, el $25 \%$ tuvo como primera manifestación TEP. Todas estas características presentó nuestra paciente.

\section{COMENTARIO}

Si bien las trombosis venosas en pediatría no son tan frecuentes como en la población adulta, constituyen un problema que debe ser reconocido, estudiado y tratado.

El SAF es una afección de gran importancia, pues la morbilidad y la mortalidad que conlleva adquieren cada vez mayor preponderancia. Así, cuando se sospecha la enfermedad en cualquier paciente, este debe ser evaluado por un equipo multidisciplinario a fin de establecer un diagnóstico oportuno y brindar tratamiento y educación adecuados.

\section{REFERENCIAS}

1. Pons M, Mendibil M, Arsich A, et al. Lupus eritematoso sistémico y síndrome antifosfolipidico. Arch Argent Pediatr 2001;99(4):354-9.

2. Pouymiró Pubillones P, Pouymiró Brooks Y, Pouymiró Brooks I.Síndrome de anticuerpos antifosfolípidos. Medisan 2012;16(3):429-44.

3. Lirola MJ, Camacho MS. Síndrome antifosfolípido. Protoc Diagn Ter Pediatr 2014;1:79-89.

4. Avcin T, Cimaz R, Silverman E, et al. Pediatric Antiphospholipid Syndrome: Clinical and Immunologic Features of 121 patients in an international registry. Pediatrics 2008;122(5):e1100-7.

5. Miyakis S, Lockshin MD, Atsumi T, et al. International consensus statement on an update of the classification criteria for definite antiphospholipid syndrome. J Thromb Haemost 2006;4(2):295-306.

6. Giannakopoulos B, Passsam F, Ioannou Y, et al. How we diagnose the antiphospholipid syndrome. Blood 2009;113(5):985-94.

7. Sobrino Castro U, Herráez Ortega I, Antolin Perez C, et al. Como realizar un informe de TEP (Angiotc de arterias pulmonares) de manera brillante. $31^{\circ}$ Congreso de la SERAM. 24-28 de mayo. Granada, España; 2012.

8. González Pascual E.Síndrome antifosfolípido en Pediatría. An Pediatr (Barc). [CD-ROM] 2005;62:361-4.

9. Hugues $G$. The antiphospholipid syndrome: ten years on. Lancet 1993;342(8867):341-4.

10. Miyakis S, Lockshin MD, Atsumi T, et al. International consensus statement on an update of the classification criteria for definite antiphospholipid syndrome (APS). J Thromb Haemostat 2006;4(2):295-306.

11. Nageswara Rao AA. Elwood K, Kaur D, et al. A retrospective review of pediatric antiphospholipid syndrome and thrombosis outcomes. Blood Coagul Fibrinolysis 2016;28(3):205-10.

12. Ma JR, Song HM, Xiao J, et al. Clinical analysis of 12 patients with pediatric antiphospholipid syndrome with pulmonary embolism. Zhonghua Er Ke Za Zhi 2017;55(1):25-9. 